\title{
Analyzing Surface Textures on Elliptical Wings
}

\section{A CFD Analysis of Stall Angles at High Angles of Attack}

\author{
Tarun Golla \\ Broad Run High School \& Academy of Science \\ Academies of Loudoun \\ Leesburg, United States
}

\author{
Adithya Muralikrishna \\ Rock Ridge High School \& Academy of Science \\ Academies of Loudoun \\ Ashburn, United States
}

\author{
Sundaram Thirukkurungudi \\ Academy of Science \\ Academies of Loudoun \\ Leesburg, United States
}

\begin{abstract}
Elliptical wings have a uniform lift distribution and thus the entirety of the wing stalls simultaneously at high angles of attack. However, elliptical wings also have a high level of maneuverability and would benefit from mitigating this stall. Stalling occurs due to the separation of airflow from the wing, known as boundary layer separation. This study tested the effect of leading-edge dimples, bumps, and longitudinal grooves on the prevention of the boundary layer separation on elliptical wings. The reference was an elliptical wing created in SolidWorks CAD software with no surface modifications. The bumps, grooves, and dimples were added using both SolidWorks and Autodesk Fusion

360. The data was then collected through Autodesk CFD to measure the lift acting on each wing. Each wing was tested at the following angles of attack: $10^{\circ}, 1^{\circ}, 2^{\circ}, 2^{\circ}, 30^{\circ}, 35^{\circ}, 4^{\circ}$. The results showed that the wing with an added bump was most effective in improving the stall angle at an approximate $15^{\circ}$ improvement relative to the reference. The groove resulted in a stall angle approximately $10^{\circ}$ greater than the reference. The dimple had a detrimental effect, stalling about $5^{\circ}$ lower than the reference. A comparison of the lift values showed that the results are statistically significantly different. We also ran a 2-Factor ANOVA on the data to determine the effect of the variables manipulated on the lift values obtained. This test shows that $90.00 \%$ of the variation in the data is linked to changes in the surface texture, indicating that the effects observed were almost entirely due to changes in surface texture.
\end{abstract}

Keywords-CFD, SolidWorks, elliptical, Fluid Dynamics, Autodesk

\section{INTRODUCTION}

There are two types of drag acting on each body moving through a compressible fluid: pressure and frictional drag. These pressures exert a force against the direction of movement at the front of the wing and with the direction of motion at the rear of the wing. Pressure differentials across the object where the pressure in front of the object is greater than that behind the object lead to what is commonly termed pressure drag. When the force from pressure that acts against the object's motion is greater than the force that pushes with the object, this net negative force is termed "pressure drag". A common technique to reduce pressure drag is to streamline the surface of the airfoil via elongating the body. While this equalizes the pressure in the leading and trailing edges of the aircraft, elongating an airfoil increases its surface area. Thus, the frictional drag force is increased greater than the corresponding amount of pressure drag force decreased.

If an object has substantial airflow separation over its surface, it is considered a blunt body. Because of this, blunt bodies are characterized by high pressure drag and minimal streamlining. In cases like a golf ball, when elongating is not practical, the same effect of streamlining can be achieved through surface features such as dimples. The dimples of the golf ball decrease the separation of the boundary layer by producing turbulent airflow. While turbulent airflow results in a greater frictional drag, by equalizing the pressure across the wing, the pressure drag is decreased.

Pressure drag is typically a product of boundary layer separation: the idea that the airflow around a wing separates to form a wake behind the body. A turbulent wake occurs beyond the point of separation, leading to pressure drag. This problem is exacerbated at higher angles of attack, during which the point of separation moves closer and closer to the tip of the airfoil. At a certain point, the boundary layer detaches, leading to stall and the airfoil having no lift [1]. By implementing technologies that could maintain the attachment of this boundary layer to the wing, the stall angle could be delayed. Additionally, because the coefficient of lift increases with angle of attack, if the boundary layer could be maintained at increasingly higher angles of attack, the airfoil would be able to reach higher coefficients of lift, creating a more efficient system.

An elliptical wing is defined as any wing for which the end segments of the planform, the top-down view of the entire wing, approximate segments of an ellipse. Elliptical wings also typically have a spanwise elliptical lift distribution in which the upward lift vectors across the wing resemble an elliptical curve. Although a spanwise elliptical lift distribution is theoretically the most efficient method of generating lift, the lift force per area, also known as the pressure distribution, is constant across the wing. Thus, if an elliptical wing stalls, the entirety of the wing stalls simultaneously [4]. Due to this, the elliptical wing stalls with little warning, resulting in a loss of control. If the stall angle of an elliptical wing is increased by 
reducing boundary layer separation, the benefits of the elliptical wings can be maintained while minimizing their drawbacks [5].

Static vortex generators, for example dimples on a golf ball, have the potential to improve lift and decrease drag. However, as these vortex generators exacerbate the turbulent wake, they also can create extra drag. Prior research has offered semi-spherical dimples as a method of reducing boundary layer separation [2].

Based on prior data, it was hypothesized that by adding surface features such as bumps, dimples, and grooves to the leading edge of an elliptical wing, boundary layer separation would decrease at any given angle of attack, resulting in a delayed stall and higher maximum lift.

\section{EXPERIMENTALDESIGN}

The wing created was a $9.5 \mathrm{~m}$ long, semi-elliptical wing with a NACA 747A315 cross-section. The leading and trailing edges of the wing are a combination of two semiellipses whose properties can each be described via the ratio $(r)$ of the length of the ellipse's semi-major axis to the length of the ellipse's semi-minor axis. The leading edge of the wing has an rof 10:1 and the trailing edge of the wing has an $r$ of 10:3.

The different surface textures tested were dimples, longitudinal grooves, and bumps. The dimples and bumps were both semi-spherical in nature and the groove was a semi-circular cut along the length of the wing. The surface textures were placed along two longitudinal lines: line 1 (L1) was $1 / 3$ the length of the wing from the wingtip and line 2 (L2) was $2 / 3$ the length of the wing from the wing tip.

There was a longitudinal groove on each of the reference lines starting from the leading edge of the wing and ending at $90 \%$ the chord length. For the bumps and dimples, one of either was placed along each line at a distance of $5 \%$ of the chord length from the leading edge of the wing.

The radius of the surface textures was proportional to the chord length of the reference line. The dimple/bump radius was $5 \mathrm{~cm}$ for L1 and $6.8 \mathrm{~cm}$ for L2 [6]. Similarly, the groove radius was $1.57 \mathrm{~cm}$ for $\mathrm{L} 1$ and $2.14 \mathrm{~cm}$ for $\mathrm{L} 2$. These values were calculated based on a proportionality statement in a prior study [3].

Once the control wing was designed in SolidWorks and the surface textures were added for each wing in Fusion 360, they were exported to Autodesk CFD for analysis. Due to this experiment's targeted application of elliptical wings in real-world conditions, the environment and testing conditions were set to mimic a drone or other such aircraft flying at an altitude of approximately 8000 meters. The analysis temperature was set to $-5{ }^{\circ} \mathrm{C}$ to account for this altitude. The test condition velocity was set to $165 \mathrm{~m} / \mathrm{s}$, the average flying speed of the General Atomics MQ-9 Reaper, a currently available unmanned military drone. At speeds of Mach 0.3 and above, models that treat air as compressible flow adhere to real-world performance better than incompressible models. Thus, compressible flow was implemented in the analysis. To mimic open-air conditions and allow for the software to account for the turbulence induced by the surface textures, we incorporated a kepsilon turbulence model into our analysis. The meshing area was defined to be 33.6 meters long, 12 meters deep, and 4.5 meters tall with increased refinement directly around the wing to allow for more accurate calculations. This was determined by Autodesk reference documents describing the process of modeling similarly turbulent and compressible flows. Prior validation models place the range of error relative to real-world conditions with these flow conditions at less than $5 \%$, lending credibility to the data. The four wings were tested at angles of attack from $10^{\circ}$ to $40^{\circ}$ in intervals of $5^{\circ}$ with the previously mentioned test conditions. Due to the unsteady nature of the solutions obtained from the differential equations, the final data contained ten replicates of each trial. This ensured that the data used for analysis was not simply the result of a false convergence or other errors. As stall angle was the primary focus of this investigation, lift values were the only measurement recorded.

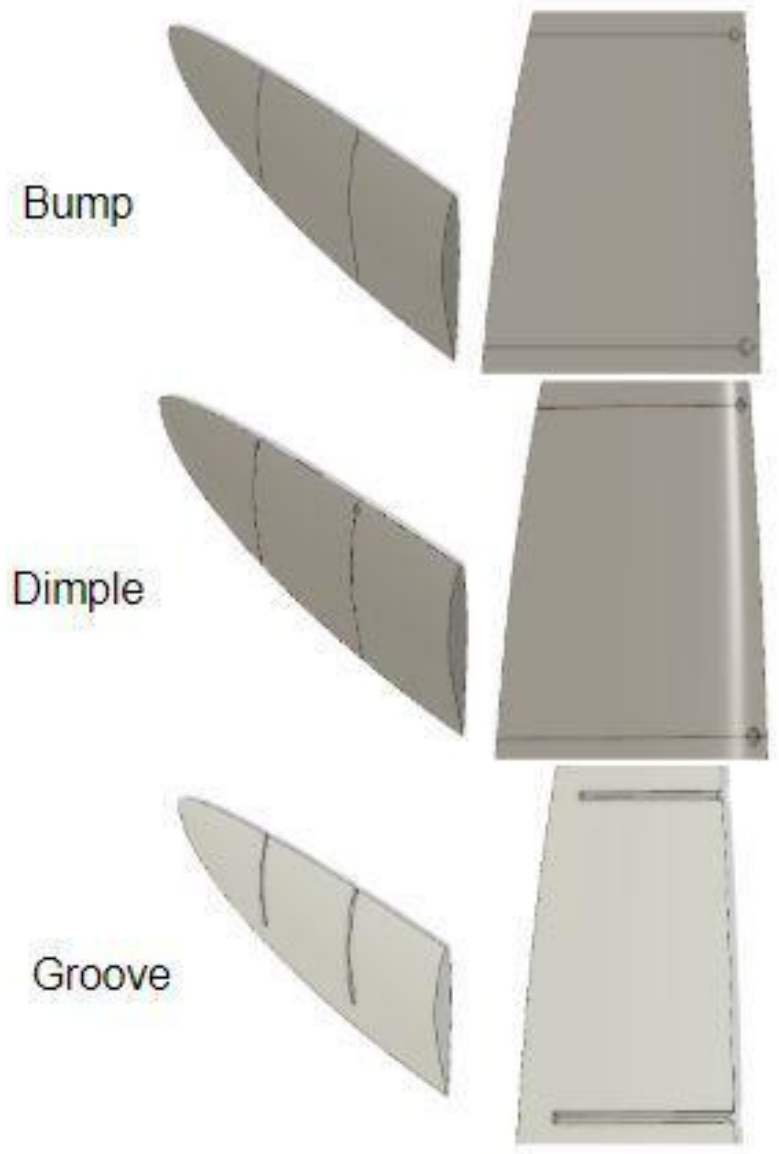

Fig. 1. Each modified wing, developed using a combination of SolidWorks and Autodesk Fusion 360, is shown above at two different perspectives. Note that the lines shown for the bump and dimple are for placement and are not surface features. 


\section{RESULTS\& DISCUSSION}

NACA 747A315: Lift Coefficient vs. Angle of Attack

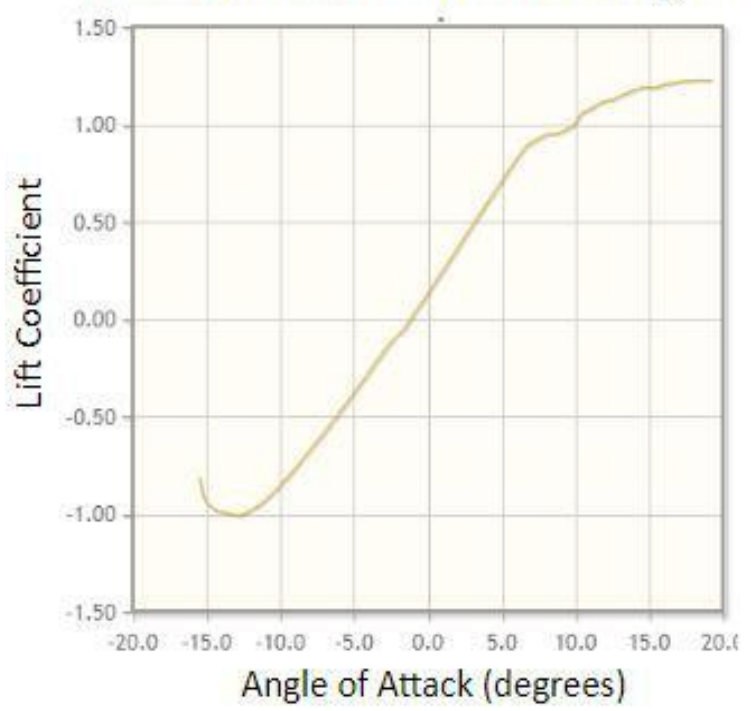

Fig. 2. NACA 747A315 lift curve.

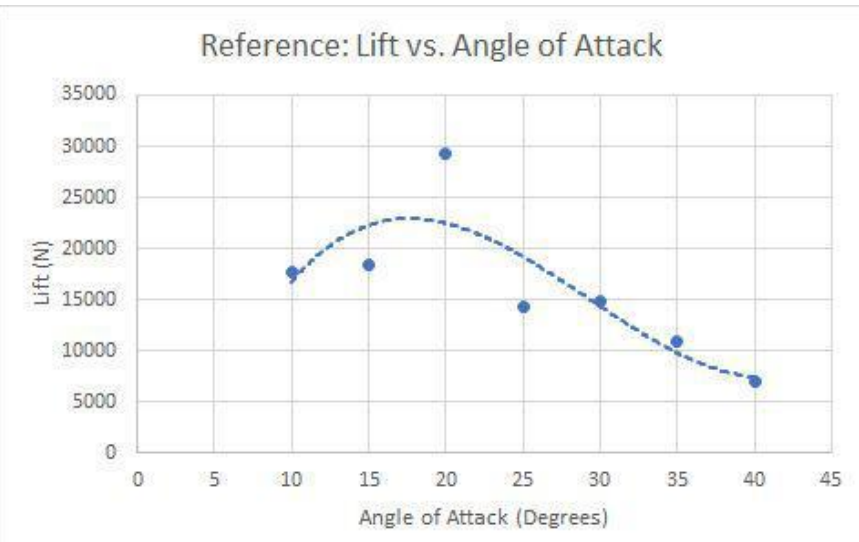

Fig. 3. Lift vs. angle of attack for the unmodified reference wing with an approximate lift curve.

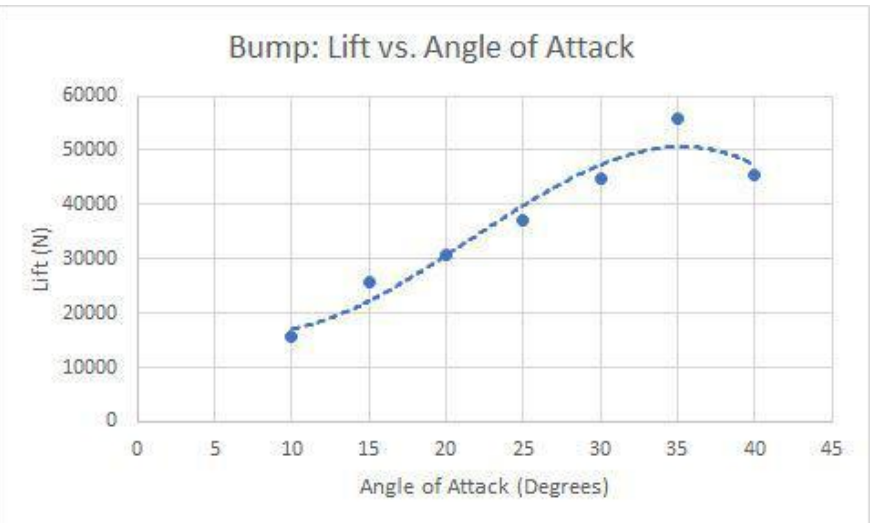

Fig. 4. Lift vs. angle of attack for the bump-modified wing with an approximate lift curve.

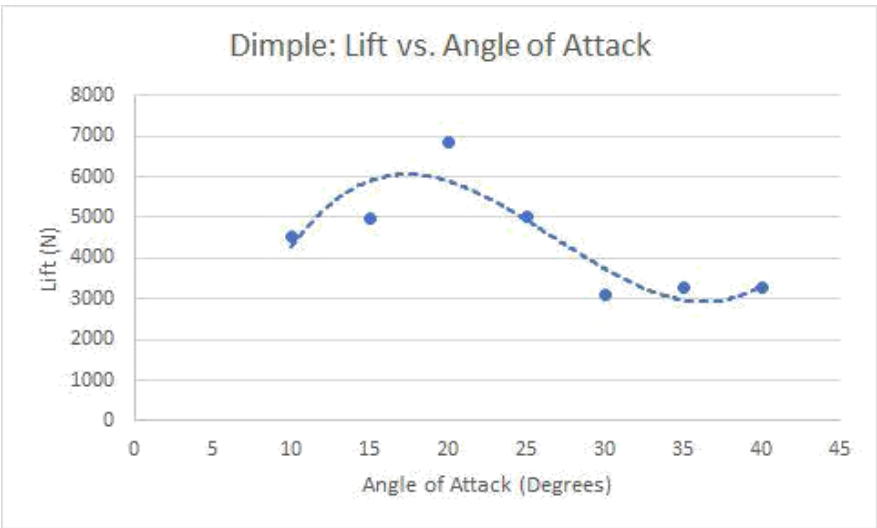

Fig. 5. Lift vs. angle of attack for the dimple-modified wing with an approximate lift curve.

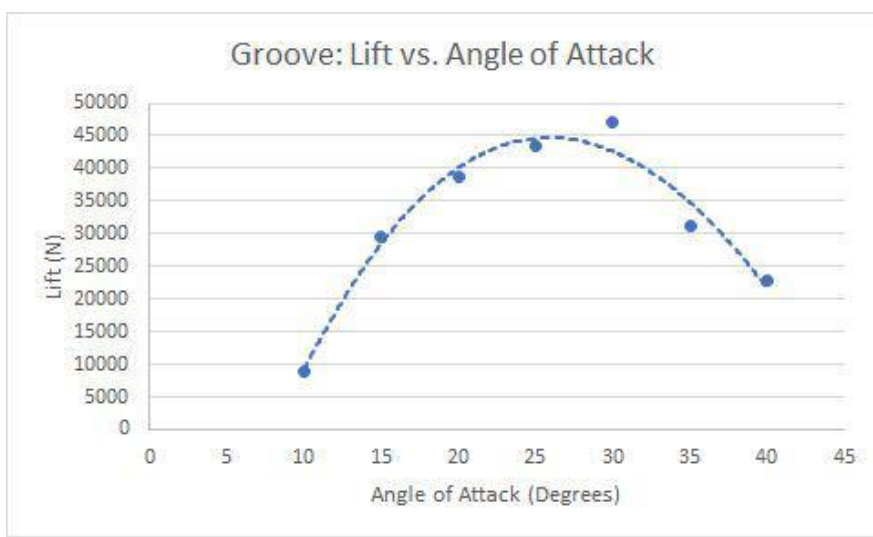

Fig. 6. Lift vs. angle of attack for the groove-modified wing with an approximate lift curve.

Due to the small sample size, 10 trials per combination, a normal distribution cannot be confirmed. Therefore, nonparametric tests are the primary statistical testing method. Kruskal-Wallis Tests were used to look for statistically significant differences between the lift values among the surface textures for each angle of attack. The test yielded an extremely significant p-value of $<0.0001$, indicating that the lift values for each wing differed significantly. The Kruskal-Wallis Tests were checked by parametric ANOVA tests. The ANOVA tests and each individual comparison were highly statistically significant suggesting that, if more data points had been obtained, the non-parametric individual comparisons would have all been highly statistically significantly different. The main parametric test used was a Two-Factor ANOVA, for which there is no nonparametric equivalent. This two-factor ANOVA allows us to see how much of the variation in the data can be attributed to changes in individual factors in the data. Based on this, the largest source of variation at $63.8 \%$ was changes in the surface texture. $10 \%$ of the variation can be attributed to changes in the angle of attack. $26.2 \%$ of the variation can be attributed to changes in both angle of attack and surface texture. $0.00001 \%$ of the variation in the data can be attributed to random error, an extremely small amount. 
Overall, the p-values obtained from both parametric and non-parametric tests were less than 0.0001, indicating an extremely low probability that the results observed were due to random sampling variance. Once this statistical result has been established, the stall angles of the wings were analyzed. These stall angles were procured from the lift curve generated by running a third-degree polynomial regression through the average lift values for each wing type. The purpose of the polynomial regression was to smooth the scatter plot which ultimately resulted in a distinguishable peak. The angle of attack that corresponds to the peak of the curve obtained via polynomial regression was considered the stall angle. Using this metric, the performance of the wings can be quantitatively analyzed by comparing their respective stall angles.

The reference wing had a stall angle of approximately $20^{\circ}$, similar to the predicted stall angle for its NACA crosssection, as seen in Figure 2. This contributes to the validation of these simulations on a real-world scale. The bump, as seen in Figure 4, showed the greatest peak lift at $55882 \mathrm{~N}$, the greatest stall angle of approximately $35^{\circ}$, and did not experience a significant decrease in lift after stall. The groove, as seen in Figure 6, initially had higher lift values than both the bump and reference airfoil but ultimately resulted in a slightly lower stall angle between $26 \mathrm{c}$ and $30^{\circ}$ and a lower peak lift of about $46960 \mathrm{~N}$. However, the dimple, as seen in Figure 5, showed significant decreases of lift on the scale of an order of magnitude at only $6825 \mathrm{~N}$ and appeared to have a lower stall angle than the reference wing of approximately $17^{\circ}$. Ultimately the hypothesis was partially affirmed as the groove and bump were capable of delaying stall and improving lift.

The quantitative analysis from the lift graphs at the beginning of this section is further supported by a qualitative analysis of the velocity heatmaps on a crosssectional plane through each wing obtained during CFD post-processing. As seen in Figure 7 and Figure 9, both the reference and dimple wings had significant boundary layer separation at a $25^{\circ}$ angle of attack. The velocity heat maps also show boundary layer separation point near the leading edge and behind the wing. These points show that both the reference and dimple wings are nearing stall or have stalled.

However, in Figure 8 and Figure 10, the bump and groove wings at the same angle of attack show little to no boundary layer separation. The airflow around these crosssections shows minimal evidence of a separated boundary layer, supporting the data that the stall angle was indeed delayed by the surface texture.

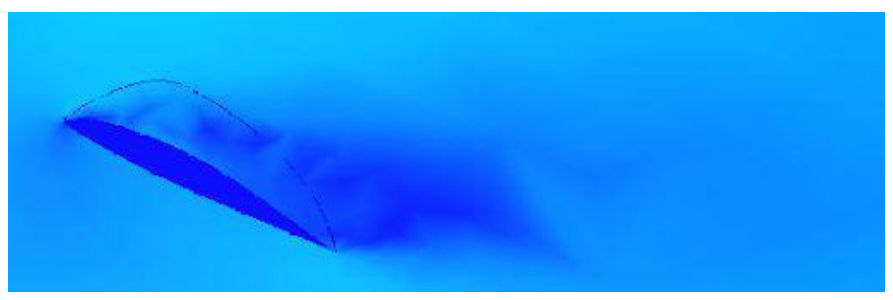

Fig. 7. Velocity heat map showing boundary layer separation behind the unmodified reference wing at a $25^{\circ}$ angle of attack.

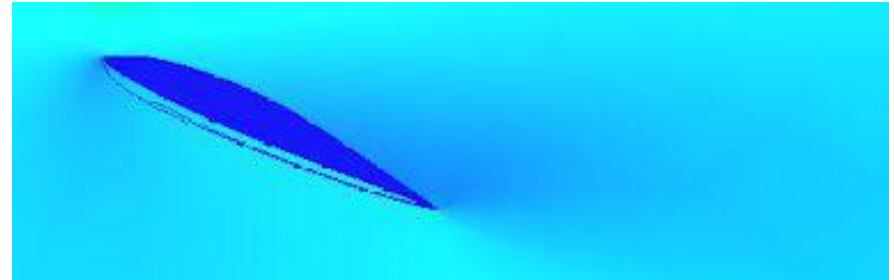

Fig. 8. Velocity heat map showing minimal boundary layer separation behind the bump-modified wing at a $25^{\circ}$ angle of attack.

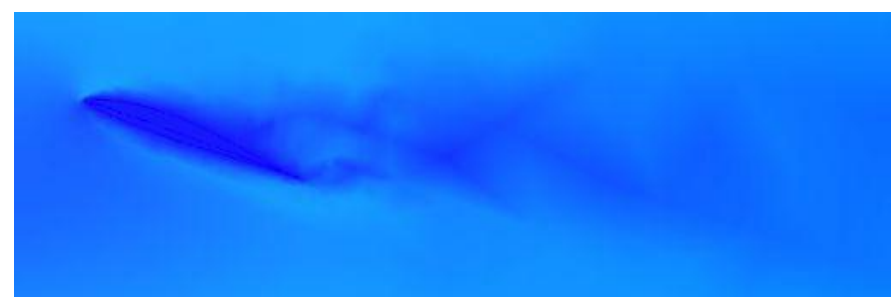

Fig. 9. Velocity heat map showing boundary layer separation behind the dimple-modified wing at a $25^{\circ}$ angle of attack.

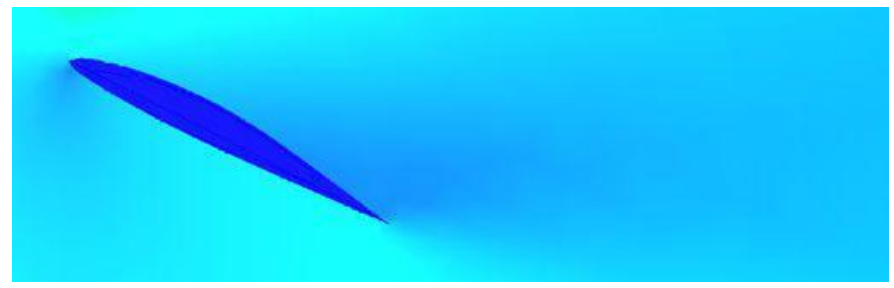

Fig. 10. Velocity heat map showing minimal boundary layer separation behind the groove-modified wing at a $25^{\circ}$ angle of attack.

\section{LIMITATIONS\& FUTUREWORK}

This research was limited by the computational power available. More refined investigations can be conducted through cloud computing services, thereby improving the computational speed and power available to the researchers. This was a preliminary exploration into stall prevention methods on elliptical wings and thus has several avenues for future expansion, the first of which is a transient analysis. In a transient flow analysis, time is a factor that can be used to understand the time-dependencies of stall on wings modified by surface textures relative to a reference wing. Secondly, the surface texture geometries, for example the radius and shape, can be modified to produce higher stall angles and lift values on elliptical wings. A wing, even if modified, must be able to handle the stress of flight, and thus a structural analysis would be paramount to such a wing's applications to industry at large. Specifically, the groove removes a significant amount of material from the wing when compared to the bump and dimple. Thus, it would be important to determine the effect on the structural integrity of the wings these surface textures have. Finally, wind tunnel testing would allow for another method of validation of our data, allowing for a better indication of how the wing would perform in real-world conditions.

\section{CONCLUSIONS}

Through this investigation, we were able to determine the effectiveness of three individual surface textures at stall prevention. Bumps and grooves were shown to be effective stall prevention mechanisms, increasing the peak stall 
angle by $50-100 \%$. However, dimples were shown to be an ineffective method of stall prevention, resulting in a significant decrease in both lift and stall angle. Evaluating the impacts of these findings, it can be seen that that elliptical wings, highly efficient yet out of use due to their stall characteristics, should be explored once again within the industry given the potential of surface textures to delay their undesired stall characteristics.

\section{ACKNOWLEDGMENT}

We would like to thank the Academy of Science, currently a subset of the Academies of Loudoun, for providing us with the faculty support and resources necessary for this research.

\section{REFERENCES}

[1] Bakker, A. (2006). Lecture 11 - Boundary layers and separation [PDF]. Retrieved from http://www.bakker.org/dartmouth06/engs150/11-bl.pdf

[2] E. Livya, G. Anitha, P. Valli (2015). Aerodynamic analysis of dimple effect on aircraft wing. World Academy of Science, Engineering and Technology International Journal of Aerospace and Mechanical Engineering, Vol:9, No:2

[3] Fernandez, E., Guha, T., \& Kumar, R. (2013). Effect of longitudinal ridges on the aerodynamic characteristics of an airfoil. AIAA Aerospace Sciences Meeting Including The New Horizons Forum And Aerospace Exposition, (51). doi: 10.2514/6.2013-489

[4] Ghadirzad, N., Mirmofidi, S. M., \& Zafarmand, B. (2011). Numerical analysis of turbulent viscous flow around an elliptical airfoil and cylinder by potential flow patterns, vortices, sources \& sinks: Random vortex method simulation. 2011 IEEE 3rd International

[5] Sun, W., Gao, Z., Du, Y., \& Xu, F. (2015). Mechanism of unconventional aerodynamic characteristics of an elliptic airfoil. Chinese Journal of Aeronautics, 28(3), 687-694. doi:10.1016/j.cja.2015.03.009

[6] Zhou, Y., \& Wang, Z. (2012). Effects of surface roughness on separated and transitional flows over a wing. AIAA Journal, 50(3), 593-609. doi: 10.2514/1.j051237 